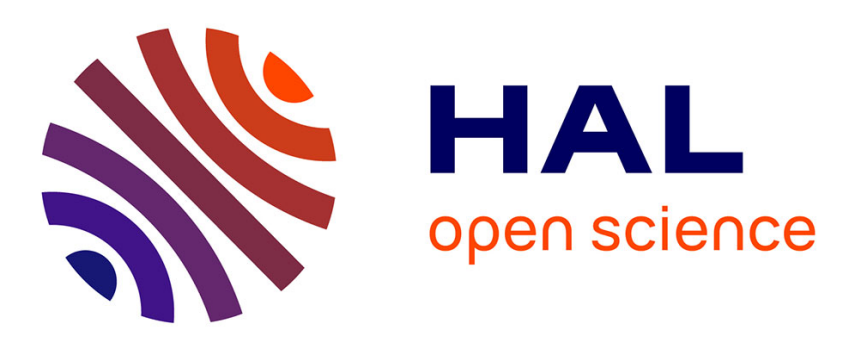

\title{
Nonlinear delayed feedback model for incompressible open cavity flow
}

\author{
F. Tuerke, F. Lusseyran, Denisse Sciamarella, L. Pastur, G. Artana
}

\section{To cite this version:}

F. Tuerke, F. Lusseyran, Denisse Sciamarella, L. Pastur, G. Artana. Nonlinear delayed feedback model for incompressible open cavity flow. Physical Review Fluids, 2020, 5 (2), 10.1103/physrevfluids.5.024401 . hal-03029730

\section{HAL Id: hal-03029730 \\ https://hal.science/hal-03029730}

Submitted on 28 Nov 2020

HAL is a multi-disciplinary open access archive for the deposit and dissemination of scientific research documents, whether they are published or not. The documents may come from teaching and research institutions in France or abroad, or from public or private research centers.
L'archive ouverte pluridisciplinaire HAL, est destinée au dépôt et à la diffusion de documents scientifiques de niveau recherche, publiés ou non, émanant des établissements d'enseignement et de recherche français ou étrangers, des laboratoires publics ou privés. 


\title{
Nonlinear delayed feedback model for incompressible open cavity flow
}

\author{
F. Tuerke $\odot,{ }^{1,}{ }^{*}$ F. Lusseyran $\odot,{ }^{2}$ D. Sciamarella $\odot,{ }^{3}$ L. Pastur, ${ }^{4}$ and G. Artana $\oplus^{1,5}$ \\ ${ }^{1}$ Laboratorio de Fluidodinámica, Facultad de Ingeniería, Universidad de Buenos Aires, \\ Avenida Paseo Colón 850, C1063ACV Buenos Aires, Argentina \\ ${ }^{2}$ LIMSI, CNRS, Université Paris-Saclay, 91405 Orsay, France \\ ${ }^{3}$ Institut Franco-Argentin d'Études sur le Climat et ses Impacts, UMI No. 3351, \\ CNRS, CONICET, UBA, C1428EGA Buenos Aires, Argentina \\ ${ }^{4}$ IMSIA, UMR No. 9219, ENSTA ParisTech, 828 Boulevard des Marechaux, 91120 Palaiseau, France \\ ${ }^{5}$ Consejo Nacional de Investigaciones Científicas y Técnicas, Godoy Cruz, 2290, \\ C1425FQB Buenos Aires, Argentina
}

(Received 22 June 2019; accepted 21 January 2020; published 20 February 2020)

\begin{abstract}
The dynamics of an oscillating shear layer when confined is enriched by retarded actions whose physical modeling is not trivial. We present a nonlinear delayed saturation feedback model, which allows us to correctly reproduce the complex shear layer spectra observed experimentally in open cavity flows in the incompressible limit. The model describes the evolution of the amplitude of the shear layer instabilities and considers two hydrodynamic feedback mechanisms directly related to the confinement introduced by the walls. One is associated with reflections of instability waves on the vertical cavity walls and the other to intracavity recirculation flow. These feedback mechanisms provide retarded actions with time lags that are used in the delay differential equation and allow the computation of the model parameters on physical grounds. The frequency components of six experimental cases in different flow regimes are well recovered by the dynamical model. The results show that the model with a single feedback mechanism produces monoperiodic oscillations of the amplitude, while the interplay of two purely hydrodynamic feedback mechanisms allow quasiperiodicity to develop.
\end{abstract}

DOI: 10.1103/PhysRevFluids.5.024401

\section{INTRODUCTION}

Open cavity flow is a prominent example of impinging shear flows, which have been subject to numerous theoretical [1-3], experimental [4-6], and numerical [7,8] studies over the past decades. Impinging shear flows are known to exhibit self-sustained oscillations and it is widely agreed upon in the literature [9] that some kind of feedback mechanism organizes the flow globally and hence is responsible for the appearance of well-defined peaks in the power spectra of the fluctuating velocity components. Reference [9] further noted that this enhanced organization is not locally confined to the region of the downstream cavity edge, but extends along the entire length of the shear layer, thereby reinforcing the concept of disturbance feedback.

In compressible flow over open cavities the feedback is commonly assumed to be of acoustic nature. The impinging shear layer creates sound waves that travel upstream, interact with the incoming shear layer, and thus close the feedback loop. This is known as the flow acoustic [10] mechanism. In the incompressible limit the wavelength of the sound wave is much greater than the

\footnotetext{
*Corresponding author: florian.tuerke@ gmail.com
} 
cavity length and other mechanisms are likely to cause the delayed feedback. Experimentally it is observed that, depending on the Reynolds number, a single frequency peak or two nonharmonic frequency peaks stand out in the shear layer spectrum $[5,8,11,12]$. Despite the fact that this last phenomenon has been known for a long time, the underlying mechanism, responsible for the two nonharmonic modes in low-Mach-number open cavity flow, remains unclear.

Reference [13] argued for a feedback mechanism in terms of nonlinear interaction between two shear layer modes, while Ref. [3] proposed to consider a compatibility condition based on the reflection of hydrodynamic instability waves from the vertical walls of the cavity. The condition is based on a more general theory of instabilities in finite domains, developed in [14], which states that after successive reflections of the instability waves on the downstream and upstream walls, the local amplitude of a given wave cannot increase in time. This leads to a quantization mechanism of the frequency band and hence explains the amplification of nonharmonic modes. Which modes are selected from the discrete set of frequencies however could not be determined by their analysis. Reference [15] very recently showed that in the squared cavity two successive bifurcations lead to two limit cycles with different frequencies and different numbers of structures which propagate along the top of the cavity and circulate in its interior. In contrast, Ref. [16] accredits the quasiperiodicity of a two-dimensional open cavity flow to the shear layer and discuss it with respect to the spectrum of the linearized Navier-Stokes operator.

Roughly a quarter century prior to that work, Ref. [17] analyzed the fluctuations of the reattachment length of a confined jet. They described a retarded hydrodynamic action, where the feedback mechanism occurs as a consequence of perturbation introduced by vortex packages that are advected upstream in the recirculation region. This hypothesis was backed in [18] and more recently in [12], which suggested that this mechanism is also present in cavity flows where the impinging shear layer feeds vorticity into the intracavity region. When oscillations are high enough, this mechanism is discontinuous and vorticity is injected in parcels. These vortex packages are transported by the intracavity flow, which, depending on the cavity aspect ratio, may adopt different flow configurations. One of them is a large downstream vortex that may be accompanied by an upstream vortex. Reference [19] showed for this last flow configuration and by means of two-dimensional numerical simulations that the vortex packages can circle in a carousel-like manner inside the downstream large vortex and explained the quasiperiodic flow regime commonly observed in many incompressible open cavity flows. This carousel flow configuration has been observed also experimentally in different works such as [20-22].

In this paper we combine the physical arguments of the reflected instability waves in [3] and the carousel model first introduced in [19] with the nonlinear delayed feedback model proposed in [17]. This approach yields a model for incompressible open cavity flows that, as we will show, correctly reproduces the experimentally measured power spectra in [21].

The paper is organized as follows. In Sec. II the flow structure, the flow regimes, and the underlying timescales of the carousel mechanism are described. In Sec. III we introduce the dynamic model, its parameters, and the involved timescales. The results are presented and discussed in Sec. IV. In Sec. V a summary is given and conclusions are drawn.

\section{FLOW DESCRIPTION}

We consider a single open cavity flow, schematically depicted in Fig. 1. The cavity has a length of $L=0.1 \mathrm{~m}$ and depth of $H$ with a cavity aspect ratio $\Gamma=L / H=2$, kept constant throughout this study. The incoming laminar boundary layer is characterized by the freestream velocity $U_{\infty}$ and the momentum thickness $\Theta_{0}$. The control parameter, which is varied to obtain different flow regimes, is the Reynolds number $\operatorname{Re}_{L}=U_{\infty} L / v$, defined in terms of the freestream velocity $U_{\infty}$, the cavity length $L$, and the kinematic viscosity $v$ of the fluid. Following [3], the effective momentum thickness $\Theta_{\text {eff }}$ is defined as the momentum thickness at the streamwise position for which a best fit (in the least-squares sense) between the experimental and the analytical hyperbolic tangent 


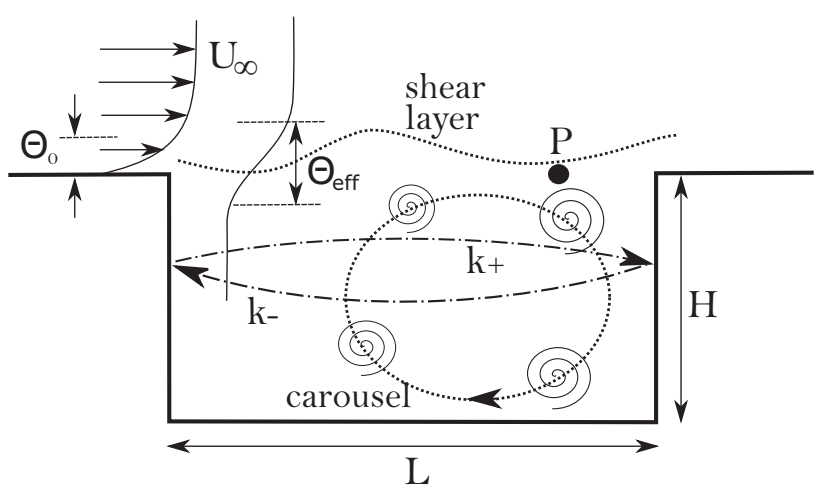

FIG. 1. Sketch of open cavity flow. Flow is from left to right, as indicated by the arrows. See the text for details.

profile is obtained. The spectral results are represented in terms of the Strouhal number, defined as $\mathrm{St}_{L}=f L / U_{\infty}$, where $f$ is the measured frequency.

\section{A. Timescales}

The incoming boundary layer separates at the cavity's leading edge and forms a shear layer that spans the cavity and impinges on the downstream cavity edge. Above a critical Reynolds number the shear layer starts to oscillate periodically at a frequency $f_{\text {shl }}=1 / T_{\text {shl }}$, where $T_{\text {shl }}$ is the shear layer timescale.

The oscillation is associated with hydrodynamic instability waves that propagate along the cavity length. Reference [3] discussed in detail the possibility and consequences of the reflection of these waves at the cavity edges. The analysis of the reflections leads to a set of discrete frequencies satisfying a compatibility condition, which explains the amplification of nonharmonic frequencies in open cavity flow. The analysis is based on a downstream traveling Kelvin-Helmholtz-like $k_{+}$wave and on an upstream traveling $k_{-}$wave, both obtained from a spatiotemporal linear stability analysis. Figure 1 shows the mechanism schematically. The $k_{-}$wave perturbs the shear layer upstream in a delayed feedback with a retardation determined by the celerity of this wave. Thus the upstream traveling $k_{-}$wave provides another timescale $\tau_{-}$that will be used in our dynamical model and discussed in detail in Sec. III C.

Upon impingement on the downstream cavity wall, the fluctuating shear layer regularly injects small vortex packages into the recirculation region, where they circumvent in a carousel-like manner and may return to and interact with the incoming shear layer, as described in detail in [19]. As was found therein, this delayed feedback mechanism is responsible for the complex composition of the shear layer spectra, commonly found in incompressible open cavity flow $[5,6,8,23]$. Figure 1 schematically depicts the carousel mechanism in the recirculation region of open cavity flows. Experimental flow visualizations of open cavity flows and other impinging shear flows that confirm the carousel mechanism can be found in various works in the literature $[5,8,17,22]$.

The circular movement in the carousel is associated with a period and in consequence a characteristic time. The turnover time of the circular motion of the recirculation region may be estimated with $T_{\mathrm{to}}=\pi H / V_{\mathrm{cr}}$, where $V_{\mathrm{cr}}$ is the carousel speed. As found in [19], the carousel speed and hence the turnover time is a function of the Reynolds number and a good approximation is $V_{\text {cr }}=0.2 U_{\infty}$.

Viscous dissipation governs the duration of the vortex packages. Their lifetime can be estimated by $T_{\mathrm{lt}} \sim \eta^{2} / \nu$, where $\eta$ is the radius of the small injected vortices and $v$ the kinematic viscosity of the fluid. The lifetime of the small vortices $T_{\mathrm{lt}}$ and the turnover time of the recirculation region $T_{\text {to }}$ 
determine if the small vortex packages may return to the shear region and perturb the shear layer, producing in this way a delayed feedback mechanism.

\section{B. Flow regimes}

As the control parameter (here the Reynolds number, even though $L / \Theta_{0}$ is also commonly used in the literature) varies, different flow regimes are observed experimentally in incompressible open cavity flow [6,8,21]. At low Reynolds numbers a steady regime occurs in which the weakness of the shear layer oscillation leads to the lack of injected vortex packages and hence to the absence of the carousel mechanism. A periodic regime, where a single frequency peak dominates the power spectrum, is reached when the shear layer starts to oscillate and as a consequence injects small vortex packages into the cavity. In this case either (i) the carousel feedback mechanism is too weak to take effect $\left(T_{\mathrm{lt}}<T_{\mathrm{to}}\right.$ ) or (ii) the carousel mechanism is strong enough $\left(T_{\mathrm{lt}} \geqslant T_{\mathrm{to}}\right)$ but synchronized with the shear layer. The periodic regime can hence be understood as the result of (i) the absence of coupling or (ii) the synchronized coupling between the linear instability and the nonlinear delayed feedback. A quasiperiodic regime is found when for $T_{\mathrm{lt}} \geqslant T_{\mathrm{to}}$ the carousel becomes desynchronized with the shear layer. Hence, the quasiperiodic regime can be understood as the result of the desynchronized coupling between the linear instability and the nonlinear delayed feedback [19]. Note that large modifications of the aspect ratio of the cavity may lead to flow configurations that do not agree well with the scenario we describe.

\section{MODEL}

\section{A. Motivation for the model}

To analyze the origin of the complex spectra of a shear layer of a cavity flow it is convenient to recall that at a given point a free shear layer oscillates with a constant amplitude. The complexity we observe in the cavity is related to the existence of a free shear layer interacting with walls in its proximity and with the intracavitary flow. This interaction introduces perturbations to the flow that extend to the region of the onset of the shear layer instability and generate a time delay.

The dynamic model we propose includes the mechanism of interaction of the shear layer with the carousel. The shear layer dynamics, however, is not only affected by this retarded action. Also the finiteness of the system and the consequential reflection of waves have to be included.

Thus the dynamical system considered here is composed of two interacting oscillators: the oscillating shear layer with reflecting walls and the vortex carousel, as schematically depicted in Fig. 1. Each system has a distinct time lag: $\tau_{1}$ is the reflecting wave time delay and $\tau_{2}$ is the carousel's time delay. If only one oscillator is considered it is not possible to generate spectra with nonharmonic peaks.

Reference [17] analyzed a confined jet and denoted by $A(t)$ the amplitude of the shear layer instability at one point of its development in the cavity. We propose here to indicate with $A(t)$ the amplitude of the shear layer instability at a point close to the impingement edge. Following [17], we propose to describe its time evolution in the form

$$
\frac{d A(t)}{d t}=r A(t)-\int_{0}^{\infty}\left[\mu\left|A\left(t-t^{\prime}\right)\right|^{2} f_{1}\left(t^{\prime}\right)+\kappa\left|A\left(t-t^{\prime}\right)\right|^{2} f_{2}\left(t^{\prime}\right)\right] d t^{\prime} A(t) .
$$

The parameter $r$ represents the usual instability growth rate, while $\mu$ and $\kappa$ stand for the sensitivities to the respective timescales $\tau_{1}$ and $\tau_{2}$. The parameter $\mu$ is associated with the shear layer, while $\kappa$ is associated with the vortex carousel. In both convolution products, the memory functions $f_{1,2}\left(t^{\prime}\right)$ modulate the time-delayed actions of the nonlinear term $\left|A\left(t-t^{\prime}\right)\right|^{2}$. The form of both memory functions can be elaborated on physical grounds. For instance, their maxima must be respectively found at $t^{\prime}=\tau_{1}$ and $t^{\prime}=\tau_{2}$ as they correspond to the mean return times of active saturating wave packets convected upstream to the cavity leading corner. These wave packets are spatially extended and may be active during a finite time $\sigma_{1,2}$, spreading around the mean return times $\tau_{1,2}$. The 
most natural shape for $f_{1,2}\left(t^{\prime}\right)$ could therefore be a Gaussian function, which naturally expresses spreading of variance $\sigma_{1,2}$ and locality around the mean return times $\tau_{1,2}$. As pointed out in [17], the spatial nature of the flow is included in the formulation of the instability dynamics via the spatial meaning of $\tau_{1,2}$ and $\sigma_{1,2}$. In the same spirit of [17], the problem can be further simplified by only considering the infinitely localized time-delayed action of the wave packets, when the spreading timescales are considered small compared to the cavity length $\sigma_{1,2} \ll \tau_{1,2}$. In such a case, $f_{1,2}\left(t^{\prime}\right)$ reduce to the Dirac $\delta$ functions $f_{1,2}\left(t^{\prime}\right)=\delta\left(\tau_{1,2}\right)$ and Eq. (1) is rewritten

$$
\frac{d A(t)}{d t}=r A(t)-\left[\mu A\left(t-\tau_{1}\right)^{2}+\kappa A\left(t-\tau_{2}\right)^{2}\right] A(t) .
$$

In the forthcoming part of the paper, we shall make use of this simplified form (2) of the model equation.

An alternative approach was proposed in [24], which considered two open flows: the wake of a cylinder and an open cavity flow. For the wake flow and when the Reynolds number is slightly above the critical Reynolds number (threshold of the vortex shedding), the authors derived from NavierStokes equation a scalar Stuart-Landau amplitude equation with three complex parameters. They showed that these parameters converge numerically to constant values when estimated considering large downstream domains (at least 50 diameters). Based on the wake flow analysis, the authors proposed to apply a similar equation to describe the dynamics of open cavity flows. In the present work we prefer to pose the amplitude equation considering a bounded domain and the physics associated with the feedback mechanisms imposed by the walls. These are expressed in Eq. (2) by the time-delay constants.

\section{B. Linear stability analysis of the amplitude equation}

Reference [17] studied the time-delayed equation (2) with $\kappa=0$ (single feedback mechanism). In that case the trivial solution $A_{0}=0$ is found to be exponentially divergent, while the fixed point at $A_{0}=\sqrt{r / \mu}$ is unstable when $r \tau_{1}>\pi / 4$. In this condition a small increment of $A(t)$ produces a monoperiodic oscillation of the value of the amplitude whose frequency is determined by nonlinear effects. Reference [17] found that the period of the oscillations, when $r \tau_{1} \geqslant \pi / 4$, is a function of $r$ and $\tau_{1}$ in the form

$$
T_{A} \simeq \tau_{1}\left(2+\frac{(1-\beta)^{2}}{1-\beta^{2}} \exp \left[2\left(1-\beta^{2}\right) r \tau_{1}\right]\right),
$$

with $\beta \simeq 0.45$. Considering this value, the period of the oscillations is $T_{A} \geqslant 3.4 \tau_{1}$. In terms of our problem, the solution at the fixed point $A_{0}$ corresponds to a condition in which the peak amplitudes of the fluctuations of the shear layer are not constant but oscillate in time around a constant value equal to $\sqrt{r / \mu}$ with a period of oscillation equal to $T_{A}$.

When Eq. (2) contains two delay times, the system exhibits three fixed points, namely, $A_{0_{1}}=0$ and $A_{0_{2,3}}= \pm \sqrt{\frac{r}{\mu+\kappa}}$. The state of the system cannot be stable as soon as $r>0$, since the evolution equation of a small increment $\delta A(t)$ around $A_{0_{1}}=0$ is, to leading order in $\delta A(t)$,

$$
\frac{d}{d t} \delta A(t)=r \delta A(t)
$$

which is for $r>0$ exponentially divergent. The other fixed points $A_{0_{2,3}}$ are unstable under special restrictions. For a small perturbation around $A_{0_{2,3}}$ we obtain, from Eq. (2), to leading order in $\delta A(t)$,

$$
\frac{d}{d t} \delta A(t)=\frac{-2 r\left[\mu \delta A\left(t-\tau_{1}\right)+\kappa \delta A\left(t-\tau_{2}\right)\right]}{\mu+\kappa} .
$$

For $\kappa=0$ we recover the results for a single delay time as considered in [17]. Applying the normal mode ansatz, we seek solutions in the form $\delta A(t)=\delta\left(A_{0} e^{s t}\right)$, where $s=\omega_{r}+i \omega_{i}$ is in 
general a complex number. From Eq. (5) we obtain

$$
\begin{aligned}
\omega_{r} & =-\frac{2 r}{\mu+\kappa}\left[\mu \cos \left(\omega_{i} \tau_{1}\right) e^{-\omega_{r} \tau_{1}}+\kappa \cos \left(\omega_{i} \tau_{2}\right) e^{-\omega_{r} \tau_{2}}\right], \\
\omega_{i} & =\frac{2 r}{\mu+\kappa}\left[\mu \sin \left(\omega_{i} \tau_{1}\right) e^{-\omega_{r} \tau_{1}}+\kappa \sin \left(\omega_{i} \tau_{2}\right) e^{-\omega_{r} \tau_{2}}\right] .
\end{aligned}
$$

Considering these equations, we can easily obtain the following set of conditions for which the system becomes unstable $\left(\omega_{r}>0\right)$ :

$$
r \tau_{1} \geqslant \frac{\pi}{4}, \quad r \tau_{2} \geqslant \frac{\pi}{4}
$$

The solution at the fixed points $A_{0_{2,3}}$ corresponds to a situation in which small increments of the amplitude $A(t)$ produce oscillations of the value of the amplitude that are not monoperiodic.

\section{Time delay for the shear layer}

Reference [3] showed, by means of a spatiotemporal linear stability analysis, that two branches of modes are involved in the selection mechanism of the self-sustained shear layer oscillations, depending on whether the wave travels forward (the so-called $k_{+}$branch) from $x=0$ to $x=L$ or backward (the $k_{-}$branch). The $k_{+}$(forward) branch is associated with Kelvin-Helmholtz-like modes, mainly shaped in the shear layer, whose celerity $c_{+}$is the shear layer mean velocity $\bar{U} \simeq U_{\infty} / 2$. The $k_{-}$(backward) branch has unusual features, since the normal modes are shaped in both the outer and the inner flow, and the celerity $c_{-}$increases with the mode frequency from values of the order of $c_{+}$at the lowest (cutoff) frequency up to the sound celerity at large frequencies. The distance between the two cavity walls selects a discrete set of allowed frequencies and imposes a condition on $\Re \Delta k=\Re\left(k_{+}-k_{-}\right)$that must be an integer multiple of $\pi / L$, according to the Ref. [14] condition.

Given the large differences between these celerities (see Figs. 11 and 12 in [3]), we observe that the wave propagating downstream has associated with it a characteristic forward time $\tau_{+} \equiv L / c_{+}$ much larger than the one of the wave propagating upstream with a backward time $\tau_{-} \equiv L / c_{-}$. In the semiempirical [10] model, the backward wave is acoustic and propagates at the speed of sound $c_{s}$ and hence $\tau_{-}=L / c_{s}$. In the incompressible limit, the acoustic waves have $\tau_{-} \equiv 0$ and the backward feedback is instantaneous.

Considering that the feedback is produced by the reflection of perturbations on the downstream wall, we have at our disposal three possible reference time delays, based on the Kelvin-Helmholtz instability with $\tau_{+}$, based on the total feedback loop with $\tau_{+}+\tau_{-}>\tau_{+}$, or based on the backward traveling wave alone with $\tau_{-}$. Only the latter time delay $\tau_{-}$appears to be consistent with the model. We will return to this point in the discussion in Sec. IV.

\section{Time delay for the inner flow}

In this section we show how the delayed action of the small vortex structures in the recirculation region is included in the model. This problem is quite similar to the confined jet flow problem with which the nonlinear saturated delayed model was tested in [17]. In that case the feedback on the incoming shear layer was produced by single turbulent packets, shed at the reattachment point of the wall and convected upstream by the backflow of the recirculation bubble. In our case, in contrast, the position of vortex shedding is fixed (downstream cavity edge) and the considered flow regimes are not turbulent. Therefore, the flow in the recirculation region is much more organized and an arrangement of a set of vortices can be established.

As mentioned in Sec. II, this feedback mechanism may produce significant effects only when the lifetime $T_{\mathrm{lt}}$ is long enough to allow these structures to return to the proximity of the shear layer. If the time $T_{\mathrm{lt}}$ is very short compared to the turnover time of the recirculation region $T_{\mathrm{to}}$, then the 
TABLE I. Model parameter values for all cases LH20-LH25. Theoretically computed values are given in parentheses.

\begin{tabular}{lccccrrrrr}
\hline \hline Case & $\operatorname{Re}_{L}$ & $U_{\infty}$ & $\Theta_{\text {eff }}$ & $r$ & \multicolumn{1}{c}{$\mu$} & \multicolumn{1}{c}{$\kappa$} & $\alpha$ & $1 / \tau_{1}$ & $1 / \tau_{2}$ \\
\hline LH20 & 15000 & 2.250 & 1.296 & $95.6(56)$ & 720.4 & 251.6 & 0.35 & $96.9(99)$ & $11.1(11)$ \\
LH21 & 14530 & 2.180 & 1.325 & $85.8(66)$ & 101.5 & 37.1 & 0.37 & $93.1(94)$ & $10.8(11)$ \\
LH22 & 12650 & 1.898 & 1.425 & $80.0(53)$ & 61.3 & 29.0 & 0.47 & $85.8(79)$ & $8.9(10)$ \\
LH23 & 11430 & 1.715 & 1.525 & $73.3(51)$ & 559.6 & 183.9 & 0.33 & $73.2(70)$ & $8.0(9)$ \\
LH24 & 9190 & 1.379 & 1.675 & $48.5(37)$ & 51.2 & 20.0 & 0.39 & $57.9(54)$ & $6.0(7)$ \\
LH25 & 6610 & 0.992 & 1.975 & $38.0(20)$ & 138.5 & 79.2 & 0.57 & $42.1(38)$ & $4.03(5)$ \\
\hline \hline
\end{tabular}

parameter $\kappa$ has to be set equal to zero in Eq. (2). In this case no quasiperiodic dynamics can be obtained for any set of parameters.

Let us now analyze the case when $T_{\text {lt }}$ is long enough to produce a feedback mechanism $(\kappa \neq 0)$. The time delay $\tau_{2}$ is calculated based on the carousel feedback mechanism introduced in [19] according to $\tau_{2}=2 \pi / \omega_{T} n_{v}$, where $n_{v}$ is the number of recirculating vortices and $\omega_{T}=V_{\mathrm{cr}} /(H / 2)$. The carousel speed is approximated by $V_{\mathrm{cr}} \approx 0.2 U_{\infty}$, as proposed in [17] and confirmed in [19]. The number of recirculating vortices depends on the lifetime of the vortex, on the angular velocity of the carousel, and on the frequency of feeding of vortex packages from the shear layer (or equivalently the frequency of oscillation of the shear layer).

\section{E. Instability growth rate and sensitivity parameters}

The temporal instability growth rate $r$ may be obtained from a spatiotemporal linear stability analysis, whose details can be consulted in [3]. The sensitivity parameters $\mu$ and $\kappa$ are calculated from the temporal growth rate $r$ and an initial estimated value $A_{g}$ of the symmetrical fixed points $A_{0_{2,3}}$, according to

$$
\mu=\frac{1}{1+\alpha} \frac{r}{A_{g}^{2}}, \quad \kappa=\frac{\alpha}{1+\alpha} \frac{r}{A_{g}^{2}},
$$

as follows from the linear stability calculations in Sec. III B. The initial estimation of $A_{g}$ is taken as the rms of the experimental time series. The parameter $\alpha=\kappa / \mu$ defines the influence of the interplay of the two feedback mechanisms. If $\alpha$ is too small, no feedback occurs, while if $\alpha$ is very high, the feedback becomes too strong. As reported in [19], this may destroy the carousel and chaotic behavior follows. Reasonable working values for $\alpha$ were found in the range $0.3 \lesssim \alpha \lesssim 0.6$. It may be of interest in some cases to improve the estimation of the values of $\alpha$ and $A_{g}$ and time constants. An optimization algorithm was therefore applied and is presented in the following section.

\section{F. Parameter estimation based on minimization of a norm}

The parameter set $\left\{r, \mu, \kappa, \tau_{1}, \tau_{2}\right\}$ is now estimated by means of an optimization algorithm, which minimizes the $\mathcal{L}_{2}$-norm of the difference between the logarithmic power spectra from the delayed feedback model and the power spectra calculated from experimental time series of the normal velocity component measured by laser Doppler velocimetry (LDV):

$$
\left\|\ln \left[\hat{A}\left(\mathrm{St}_{L}\right)\right]-\ln \left[\hat{v}\left(\mathrm{St}_{L}\right)\right]\right\|_{2} \text {. }
$$

In order to put the emphasis on main shear layer peaks, the Strouhal numbers are restricted to $0.3<\mathrm{St}_{L}<5$, according to the time series considered. The values calculated in the preceding section, given in parentheses in Table I, are used as initial conditions for the minimization algorithm. When the time series are long enough to satisfy the frequency resolution requirement and yield a converged statistics, a good estimator of the power spectrum is given by Welch's method. Recalling 
that the norm has to be computed at each iteration of the minimization, we have used instead an estimator based on the Yule-Walker autoregressive method, which allows a fast and good estimation of the power spectral density even computed on short time series.

The convergence to a good minimum is obtained by taking into account the constraints imposed by the fixed point relationship (8) between the parameters $r, \mu$, and $\kappa$. We consider solutions with values of $r$ close to those provided by the instability analysis. The four parameters $\alpha, A_{g}, \tau_{1}$, and $\tau_{2}$ are given at the end of the minimization process. The search for the minimum of the norm is accomplished using a MATLAB function based on the Nelder-Mead simplex direct search, while Eq. (2) is solved using the MATLAB function dde 23 for delay differential equations with constant delays.

\section{RESULTS AND DISCUSSION}

\section{A. Results of parameter estimation}

Based on the physical arguments outlined in Sec. III, the model parameters were calculated for six cases whose spatiotemporal linear stability properties were investigated in the same geometry in [3]. The optimization algorithm from Sec. III F was subsequently applied to estimate $\alpha$ and refine the remaining three parameters $r, \tau_{1}$, and $\tau_{2}$.

The theoretical predictions, together with the values adopted by the optimization algorithm (cf. Sec. III F), are summarized in Table I. They are in good agreement and are, except for the growth rate $r$, only slightly adjusted by the optimization algorithm. The theoretically predicted growth rates from the inviscid spatiotemporal stability analysis [3] are in all cases within the same order of magnitude, but up to $90 \%$ lower than the optimized values.

In all cases the values of the adapted parameters comply with the condition obtained from the linear stability analysis in Sec. III B, according to which the system becomes unstable for $r \tau_{1}>\pi / 4$ and $r \tau_{2}>\pi / 4$, respectively. Exemplary for the case LH21, the calculations of the parameters are detailed in the Appendix.

\section{B. Comparison to experimental data}

We will now compare the results obtained from the nonlinear delayed feedback model with experimental data from [5,6]. Figures 2(a)-2(f) show the experimentally measured time series of the streamwise velocity component $v(t)$ from [5,6]. Figures $2(\mathrm{~g})-2(\mathrm{l})$ depict the time series of the instantaneous amplitude of the shear layer instability $A(t)$, obtained from the nonlinear delayed feedback model. Figures 2(m)-2(r) compare the power spectra, computed from both time series, using Welch's method. The proposed model successfully reproduces the amplified frequencies of the different experimental cases, as can be seen from Figs. 2(m)-2(r). The dominant frequency peaks of both the quasiperiodic cases (LH20-LH23 and LH25) and the periodic case (LH24) are correctly reproduced by the cavity model. At first glance the model's result of the case LH25 seems to amplify too many peaks. A closer look at the experimental spectrum in Fig. 2(r) however shows that most of the predicted peaks are actually present in the experimental data. While some peaks only slightly emerge from the background, a few others are probably lost within the background level.

The main difference between the experimental spectra and the analytic spectra is concerned with the hierarchy of the different harmonics. In particular, the model tends to amplify the subharmonic $f_{2} / 2$ of the nonharmonic peak somewhat stronger, while experimentally the peak at $f_{2}$ is more amplified. This is in agreement with the two-dimensional numerical simulations in [19], where the same behavior was observed. On these grounds it may be assumed that a physical process, most likely related to spanwise dynamics and therefore not included in our model, is responsible for shifting the energy from $f_{2} / 2$ to $f_{2}$. Unsurprisingly, the background levels are very different because the dynamics of the model is much simpler and less noisy than the actual three-dimensional cavity flow dynamics. Also, the low-frequency content, which, as shown in [25], results from a 
(a)

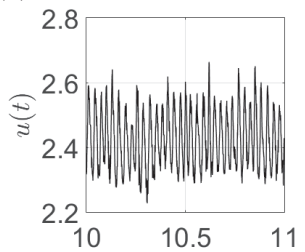

(b)

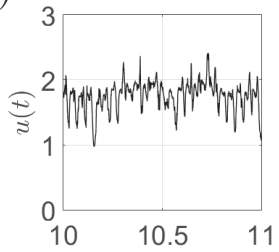

(c)

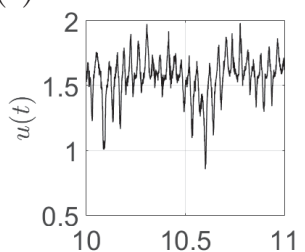

(d)

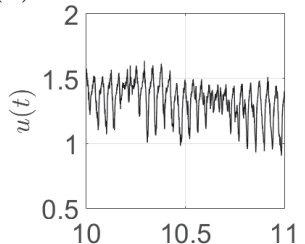

(e)

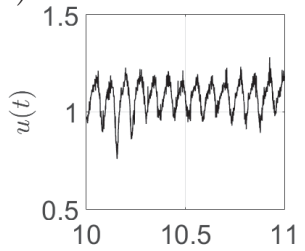

(f)

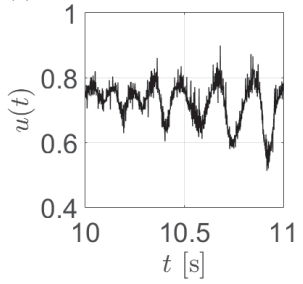

(g)

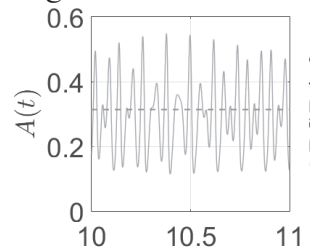

(h)

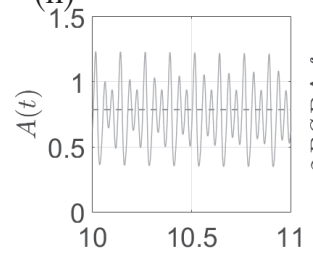

(i)

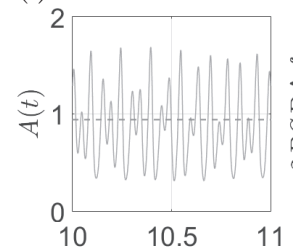

(j)

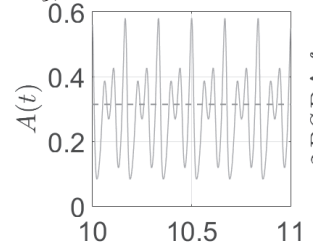

(k)

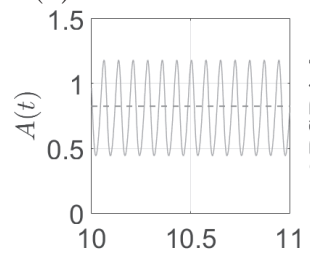

(l)

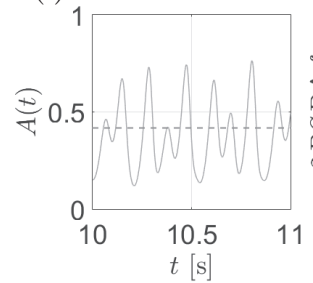

(m)

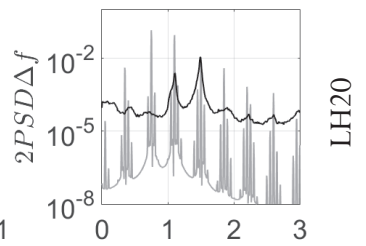

(n)

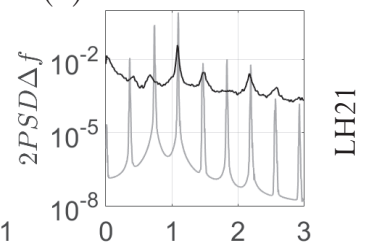

(o)

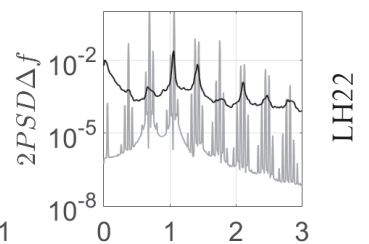

(p)

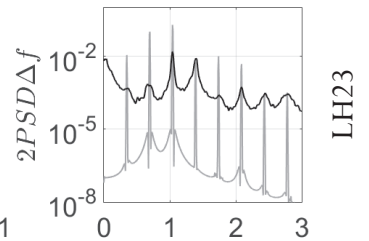

(q)

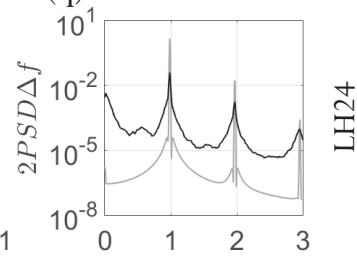

(r)

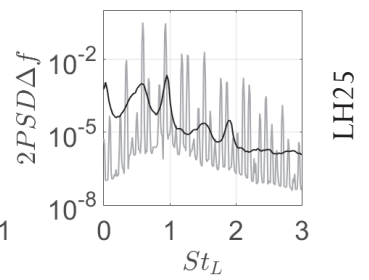

FIG. 2. Results for all cases in Table I. (a)-(f) Time series of LDV velocity measurements in the real flow. (g)-(l) Time series from the nonlinear delayed feedback model. (m)-(r) Comparison of experimental power spectra (black curve), computed from the time series in (a)-(f), and power spectra from the delayed feedback model (gray curve), computed from the time series in (g)-(l), for all cases in Table I. Horizontally dashed lines in (g)-(l) indicate the value of the stable fixed point. 
nonlinear interaction between the shear layer and the spanwise dynamics, is not present in the model's power spectrum. The two-dimensional flow we consider does not allow for the development of spanwise inner-flow structures, whose characteristic timescales are slow compared to the shear layer oscillations. The resulting low-frequency components modulate the dominant frequencies and significantly enlarge the otherwise thin peaks associated with the shear layer oscillations. These inner-flow structures develop at Reynolds numbers much smaller than the onset of the shear layer oscillations. As a consequence, experimental spectra of activated three-dimensional cavity flow always exhibit broad peaks in the power spectrum.

As mentioned in Sec. III C, the value of $\tau_{-}$provides the appropriate reference time for the time delay $\tau_{1}$. This means, therefore, that $\tau_{1}$ characterizes the upstream traveling hydrodynamic instability wave $k_{-}$and hence the feedback of the reflected shear layer instability. It is the hydrodynamic equivalent of the acoustic wave from the Rossiter mechanism. This is a different viewpoint on the cavity problem, made possible by the spatiotemporal linear stability analysis in a finite domain by [3]. The second delay time $\tau_{2}$ is motivated by the hydrodynamic feedback of the recirculating vortices (carousel feedback mechanism) first proposed in [19]. This second delayed feedback was found to be indispensable for quasiperiodic motion to be obtained from the nonlinear delayed feedback model. On the other hand, a periodic flow regime can still be observed even if the carousel feedback is present $(\kappa \neq 0$ or for that matter $\alpha \neq 0)$. The case LH24 exemplifies such a scenario. The dynamics of the shear layer and the carousel synchronize and using the terminology of [19], the ratio of the characteristic timescales of both phenomena yield $T_{\mathrm{to}} / T_{\mathrm{shl}}=p / q$, with $p, q \in \mathbb{N}$. Note that in the present model we only consider the characteristic feedback delay times and therefore there is no reason to take $T_{\mathrm{shl}}$ for $\tau_{1}$. Additional evidence that in the periodic case LH24 the carousel is not absent but only locked in with the shear layer is that for the lower-Reynolds-number case LH25 a quasiperiodic motion is recovered. The value of $\alpha$, i.e., the parameter, which governs the influence of the carousel mechanism, however, is of the same order of magnitude $(0.3<\alpha<0.6)$ in all cases, which supports the hypothesis that for the periodic case the carousel mechanism is active but locked in with the shear layer.

\section{Rossiter's mechanism revisited}

As mentioned in the Introduction, in compressible flows over cavities the commonly accepted feedback mechanism is of acoustic type, as proposed by Rossiter in [10]. That semiempirical model assumes a feedback loop between the vortex shedding and the acoustic radiation which is created upon impingement of the vortices on the downstream cavity edge. The acoustic radiation travels upstream and initiates the vortex shedding at the leading edge of the cavity, thus closing the feedback loop. Rossiter's mechanism, however, is not uncontroversial in the literature and in recent years the importance of the acoustic feedback at very high Mach numbers (supersonic flows) as well as very low Mach numbers (incompressible limit) has been debated. Reference [26] tried to improve Rossiter's original formula by adding additional terms, while Ref. [27] investigated high-Mach-number cavity flows and found that, by breaking the acoustic feedback loop, the good agreement between the measured and the predicted frequencies at high-Mach-number speeds is largely coincidental and does not reflect the correct modeling of the flow physics. On the other hand, approaching the incompressible limit, Ref. [28] tried to link the Rossiter mechanism to the results of a global instability analysis. Those results, however, showed only limited success in reproducing experimental data. At such low Mach numbers the acoustic wavelength is much greater than the cavity length and while nonharmonic cavity oscillations prevail, it is unlikely that the flow acoustic feedback continues being responsible for them. This hypothesis is backed in [29] and more recently in [21], both of which conducted cavity flow experiments in water and thus confirmed the existence of cavity edge tones in an essentially incompressible medium.

However, even in the incompressible limit we observe a highly organized flow with well-defined frequency peaks, which leads us to assume that some kind of feedback mechanism must still be active. Considering the encouraging results of the present work, together with the findings of 
previous works such as $[3,5,8,17,19]$, we suggest the feedback in incompressible open cavity flow to be of purely hydrodynamic nature. The equivalent of Rossiter's upstream traveling sound wave are the reflected instability wave $k_{-}$, which is characterized in our model by the delay time $\tau_{1}$, and the vortex carousel, characterized in our model by the delay time $\tau_{2}$.

\section{CONCLUSION}

We proposed a mathematical model to account for the different flow regimes commonly observed in incompressible cavity flows. The nonlinear delayed feedback model proposed in [17] was reformulated using a double instead of a single feedback mechanism. Two time delays were introduced, one for the shear layer associated with the reflection of hydrodynamic instability waves and one for the inner flow associated with the carousel mechanism, in accordance with previous experimental and theoretical studies. We considered experimental data of open cavity flows in a wide range of Reynolds numbers of the incompressible limit, corresponding to six experiments performed in [5,6], and obtained the parameters of the model for each case. Periodic as well as quasiperiodic regimes were retrieved, leading to power spectra whose dominant frequency peaks are in good agreement with experimental data in all flow regimes. The results confirm the interaction and importance of both the carousel feedback mechanism and the reflection of instability waves in impinging shear flows in the incompressible limit, neither of which were included in Rossiter's flow acoustic feedback mechanism.

The nonlinear double delayed feedback model we proposed offers both a coherent explanation of the phenomenon and a mathematical tool to study its dynamics, and hence further improves our understanding of open cavity flows in particular and of impinging shear flows in general.

\section{ACKNOWLEDGMENTS}

We acknowledge support from CONICET (Argentina) under Grant No. 3303, UBACYT under Grant No. 20020130100708BA, ANPCYT under Grant No. PICT 2015-2991, Science-Accueil d'Université Paris-Sud, LIA-PMF/FMF, and Project No. 13STIC-08-P-MVP of the SticAmSud program.

\section{APPENDIX: EXEMPLARY CALCULATION OF MODEL PARAMETERS}

The main focus of this article is on the physics involved in the feedback mechanisms and not in finding the more adequate methodology of model parameter estimation. We will just show here, for some exemplary case (LH21), how parameters can be estimated from experimental or numerical flow data with a less refined approach. We illustrate these aspects, taking into account results from [3]. Note that article nondimensionalized the governing equations with the mean velocity $\bar{U}=0.5 U_{\infty}$ and the effective momentum thickness $\Theta_{\text {eff }}$. The power spectrum shows a dominant frequency at $f_{1}=23.6 \mathrm{~Hz}$ for which the temporal growth rate

$$
r=-\hat{\omega}_{i} \bar{U} / \Theta_{\mathrm{eff}}=66
$$

is calculated from the value of $\hat{\omega}_{i}=-0.08$ (cf. Fig. 9 in [3]), $\bar{U}=1.09 \mathrm{~m} / \mathrm{s}$, and $\Theta_{\text {eff }}=1.325 \mathrm{~mm}$. Note that the negative sign arises from the Cauchy-Riemann relations, which lead to the [30] transformation.

As mentioned in Sec. III C, the backward traveling $k_{-}$wave must be considered to determine the time delay $\tau_{1}$. There are two possible options for calculating $\tau_{1}$. The first option is to calculate it according to

$$
\tau_{1}=L / c_{-} \bar{U}=\frac{1}{109},
$$

where $L=0.1 \mathrm{~m}$ is the cavity length and $c_{-} \approx 10$ is the nondimensional celerity of the upstream traveling $k_{-}$wave (cf. Fig. 12 in [3]). The second option is to calculate $\tau_{1}$ with the help of Eq. (3) 
as proposed by [17]. The period of the dominant oscillation is then approximated by $T \approx 4 \tau_{1}$ as discussed in Sec. III C. With the expected dominant frequency peak at $f_{1}=1 / T$, this yields

$$
\tau_{1} \approx 1 / 4 f_{1}=\frac{1}{94} .
$$

Both methods of calculating $\tau_{1}$ agree within $10 \%-20 \%$, depending on the case, hence confirming the correct choice of the reference time $\tau=\tau_{-}$we made in Sec. III C. In Table I the value of $\tau_{1}$ according to the second method is given. As outlined in Sec. III D, the time delay $\tau_{2}$ is calculated based on the carousel feedback mechanism. We obtain

$$
\tau_{2}=2 \pi / \omega_{T} n_{v}=\frac{1}{11},
$$

where $n_{v}=4$ is the number of recirculating vortices and $\omega_{T}=V_{\mathrm{cr}} /(H / 2)=0.2 U_{\infty} 4 / L$ defines the carousel rotation speed as given in [19]. Using the calculated values as the starting point, the remaining parameters were obtained from the optimization algorithm, described in Sec. III F.

[1] E. M. Lifshitz and L. P. Pitaevskii, in Physical Kinetics, Course of Theoretical Physics Vol. 10 (Pergamon Press, Oxford, 1981), pp. 281-283.

[2] P. Huerre and P. Monkewitz, Absolute and convective instabilities in free shear layers, J. Fluid Mech. 159, 151 (1985).

[3] F. Tuerke, L. Pastur, D. Sciamarella, F. Lusseyran, and G. Artana, Frequency selection mechanism in incompressible open cavity flow via reflected instability waves, Phys. Rev. E 91, 013005 (2015).

[4] A. Michalke, On the inviscid instability of the hyperbolic tangent velocity profile, J. Fluid Mech. 19, 543 (1964).

[5] J. Basley, L. Pastur, F. Lusseyran, T. Faure, and N. Delprat, Experimental investigation of global structures in an incompressible cavity flow using time-resolved PIV, Exp. Fluids 50, 905 (2011).

[6] F. Lusseyran, L. Pastur, and C. Letellier, Dynamical analysis of an intermittency in open cavity flow, Phys. Fluids 20, 114101 (2008).

[7] C. W. Rowley, T. Colonius, and A. J. Basu, On self-sustained oscillations in two-dimensional compressible flow over rectangular cavities, J. Fluid Mech. 455, 315 (2002).

[8] F. Tuerke, L. Pastur, D. Sciamarella, F. Lusseyran, and G. Artana, Experimental study of double cavity flow, Exp. Fluids 58, 76 (2017).

[9] D. Rockwell and C. Knisely, The organized nature of flow impingement upon a corner, J. Fluid Mech. 93, 413 (1979).

[10] J. E. Rossiter, Wind-tunnel experiments on the flow over rectangular cavities at subsonic and transonic speeds, Aeronautical Research Council Report No. 3438, 1966.

[11] M. Gharib and A. Roshko, The effect of flow oscillations on cavity drag, J. Fluid Mech. 177, 501 (1987).

[12] A. Maurel, P. Ern, B. Zielinska, and J. Wesfreid, Experimental study of self-sustained oscillations in a confined jet, Phys. Rev. E 54, 3643 (1996).

[13] S. Cherubini, J.-C. Robinet, P. De Palma, and F. Alizard, The onset of three-dimensional centrifugal global modes and their nonlinear development in a recirculating flow over a flat surface, Phys. Fluids 22, 114102 (2010).

[14] A. Kulikowskii, On the stability of homogeneous states, J. Appl. Math. Mech. 30, 148 (1966).

[15] Y. Bengana, J.-C. Loiseau, J.-C. Robinet, and L. S. Tuckerman, Bifurcation analysis and frequency prediction in shear-driven cavity flow, J. Fluid Mech. 875, 725 (2019).

[16] C. Leclercq, F. Demourant, C. Poussot-Vassal, and D. Sipp, Linear iterative method for closed-loop control of quasiperiodic flows, J. Fluid Mech. 868, 26 (2019).

[17] E. Villermaux and E. Hopfinger, Effect of aspect ratio on the three dimensional global instability analysis of incompressible open cavity flows, Physica D 72, 230 (1994).

[18] A. Maurel, V. Pagneux, and J. Wesfreid, Mean-flow correction as non-linear saturation mechanism, Europhys. Lett. 32, 217 (1995). 
[19] F. Tuerke, L. Pastur, Y. Fraigneau, D. Sciamarella, F. Lusseyran, and G. Artana, Nonlinear dynamics and hydrodynamic feedback in two-dimensional double cavity flow, J. Fluid Mech. 813, 1 (2017).

[20] C. Haigermoser, L. Vesely, and M. Onorato, in Proceedings of the 26th Congress of the International Council of the Aeronautical Sciences, Anchorage, 2008, edited by I. Grant (ICAS, Brisbane, 2008).

[21] J. Basley, An experimental investigation on waves and coherent structures in a three-dimensional open cavity flow, Ph.D. thesis, Université Paris-Sud, 2012.

[22] F. Tuerke, Flow in a channel with two facing cavities, Ph.D. thesis, Universidad de Buenos Aires and Univertsité Paris-Saclay, 2017.

[23] L. R. Pastur, F. Lusseyran, T. M. Faure, Y. Fraigneau, R. Pethieu, and P. Debesse, Quantifying the nonlinear mode competition in the flow over an open cavity at medium Reynolds number, Exp. Fluids 44, 597 (2008).

[24] D. Sipp and A. Lebedev, Global stability of base and mean flows: a general approach and its applications to cylinder and open cavity flows, J. Fluid Mech. 593, 333 (2007).

[25] J. Basley, L. R. Pastur, N. Delprat, and F. Lusseyran, Space-time aspects of a three-dimensional multimodulated open cavity flow, Phys. Fluids 25, 064105 (2013).

[26] H. Heller and D. Bliss, Proceedings of the 2nd Aeronautics Conference, Hampton, 1975 (AIAA, Reston, 1975), paper 1975-491.

[27] O. Unalmis, N. Clemens, and D. Dolling, Cavity oscillation mechanisms in high-speed flows, AIAA J. 42, 2035 (2004).

[28] S. Yamouni, D. Sipp, and L. Jacquin, Interaction between feedback aeroacoustic and acoustic resonance mechanisms in a cavity flow: A global stability analysis, J. Fluid Mech. 717, 134 (2013).

[29] C. B. Burroughs and D. R. Stinebring, Cavity flow tones in water, J. Acoust. Soc. Am. 95, 1256 (1994).

[30] M. Gaster, A note on the relation between temporally-increasing and spatially-increasing disturbances in hydrodynamic stability, J. Fluid Mech. 14, 222 (1962). 